\title{
Determination of ChPT low energy constants from a precise description of $\pi-\pi$ scattering threshold parameters
}

\author{
Jenifer Nebreda* \\ Departamento de Física Teórica II, Universidad Complutense de Madrid, \\ Helmholtz-Institut für Strahlen- und Kernphysik (Theorie), Universität Bonn \\ E-mail: jenifer.nebreda@fis.ucm.es
}

\section{José Ramón Peláez}

Departamento de Física Teórica II, Universidad Complutense de Madrid

E-mail: jrpelaezefis.ucm.es

\section{Guillermo Ríos}

Departamento de Física Teórica II, Universidad Complutense de Madrid, Departamento de Física. Universidad de Murcia

E-mail: griosmarefis.ucm.es

\begin{abstract}
We report on our recent results in the study of the determination of the values of the one and two loop low energy constants appearing in the Chiral Perturbation Theory calculation of pionpion scattering. For this we use a recent and precise sum rule determination of scattering lengths and slopes that appear in the effective range expansion. In addition we provide new sum rules and the values for these coefficients up to third order in the expansion. Our results when using only the scattering lengths and slopes of the S, P, D and F waves are consistent with previous determinations, but seem to require higher order contributions if they are to accommodate the third order coefficients of the effective range expansion.
\end{abstract}

Sixth International Conference on Quarks and Nuclear Physics

April 16-20, 2012

Ecole Polytechnique, Palaiseau, Paris

\footnotetext{
* Speaker.
} 


\section{Introduction}

As is well known, the strong interactions at very low energy can be studied using an effective theory for QCD for which the degrees of freedom are not the quarks and gluons, but the Goldstone bosons of the spontaneous $S U(2)$ chiral symmetry breaking, the pions. This theory is known as Chiral Perturbation Theory (ChPT) [1,2] and is built as the most general low energy expansion in powers of the pion mass and momenta that is compatible with the symmetry constraints of QCD. The details of the underlying dynamics are encoded in a set of low energy constants (LECs), which multiply the independent terms that appear in the Lagrangian. All loop divergences appearing in a calculation up to a given order can be reabsorbed by renormalization of the LECs up to that order. In this way calculations are rendered finite and scale independent to any given order of the expansion.

Only certain combinations of LECs appear in $\pi \pi$ scattering up to a given order. To leading order $O\left(p^{2}\right)$ there are no LECs. To next to leading order (NLO), or $O\left(p^{4}\right)$, which corresponds to a one-loop calculation, only four LECs, called $l_{1}, l_{2}, l_{3}$ and $l_{4}$ appear in the amplitude. To next to next to leading order (NNLO), or $O\left(p^{6}\right)$, six possible independent terms appear [3] multiplied by six constants, $\bar{b}_{i}$ with $i=1 \ldots 6$, which can be reexpanded in powers of the pion mass in terms of the four one-loop $l_{k}$ and six new NNLO LECs, denoted by $r_{i}$ [4].

Concerning the $O\left(p^{4}\right)$ LECs, we refer the reader to [5] for a recent compilation of lattice QCD and to [6] for some other estimates from quark-model-like calculations. It is worth noticing that the bulk of their values can be explained by the effect of integrating out heavier resonances and, actually, seems to be mainly saturated by the vector multiplets [7]. Some estimates from resonance saturation have also been obtained for the $O\left(p^{6}\right)$ parameters [4]. However, since perturbative QCD cannot be applied at very low energies, it is particularly difficult to obtain the values of these LECs from first principles and, with few exceptions, the LECs have been determined best from the comparison with experiment $[2,8,9,10]$.

In our work [11], which we report here, we use a very recent dispersive analysis of data in [12], which includes the latest very precise and reliable results on $K_{e 4}$ decays from the NA48/2 collaboration [13], in order to determine the values of the $O\left(p^{4}\right)$ and $O\left(p^{6}\right)$ LECs that appear in the $\pi \pi$ scattering amplitude. We will obtain the LECs from fits to the coefficients of the momentum expansion of the amplitude around threshold, usually known as the effective range expansion. The coefficients of this expansion, even up to third order, are also becoming reachable for lattice calculations, although still only limited to the highest isospin channels [14].

\section{Threshold parameters}

\subsection{Notation}

The amplitude for $\pi \pi$ scattering is customarily decomposed in terms of partial waves $t_{\ell}^{I}$, of definite isospin $I$ and angular momentum $\ell: t_{\ell}^{I}(s)=\frac{1}{64 \pi} \int_{-1}^{1} T^{I}(s, t, u) P_{\ell}(\cos \theta) d(\cos \theta), \theta$ being the scattering angle, $P_{\ell}$ the Legendre polynomials, $s, t, u$ the usual Mandelstam variables and $T$ the amplitude. With this normalization, the effective range expansion can be written as:

$$
\frac{1}{M_{\pi}} \operatorname{Re} t_{\ell}^{I}(s)=p^{2 \ell}\left(a_{\ell I}+b_{\ell I} p^{2}+\frac{1}{2} c_{\ell I} p^{4}+\ldots\right),
$$


where the $a_{\ell I}$ are usually called scattering lengths, the $b_{\ell I}$ slope parameters, the $c_{\ell I}$ shape parameters, and all of them, generically, threshold parameters.

\subsection{Threshold parameters from sum rules}

For the experimental determination of the threshold parameters we use the parametrizations of [12], which were obtained by highly constraining the data fits to satisfy three sets of dispersion relations within uncertainties. In that work, the values of the $a$ and $b$ threshold parameters were provided for all the combinations of $I$ and $\ell$ allowed by Bose symmetry, up to $\ell=3$. With the aim of minimizing the uncertainties, they were obtained from sum rules, with the only exception of the $5 a_{S 0}+2 a_{S 2}$ combination, which is orthogonal to the one appearing in the Olsson sum rule (note the spectroscopic notation, where the $\ell=0,1,2,3 \ldots$ are called S,P,D,F... waves).

Those results provided us with 12 observables determined from experiment. Moreover, we provide the calculation of the third order coefficient $c$ of the effective range expansion, which adds five more observables for the fit.

For the $c$ parameters with $\ell>0$, we use the Froissart-Gribov sum rules:

$$
\begin{aligned}
c_{\ell I}=\frac{\sqrt{\pi} \Gamma(\ell+1)}{M_{\pi} \Gamma(\ell+3 / 2)} \int_{4 M_{\pi}^{2}}^{\infty} d s\{ & \frac{16 \operatorname{Im} F^{I^{\prime \prime}}\left(s, 4 M_{\pi}^{2}\right)}{\left(s-4 M_{\pi}^{2}\right)^{2} s^{\ell+1}} \\
& \left.-8(\ell+1) \frac{\operatorname{Im} F^{I^{\prime}}\left(s, 4 M_{\pi}^{2}\right)}{\left(s-4 M_{\pi}^{2}\right) s^{\ell+2}}+\frac{\operatorname{Im} F^{I}\left(s, 4 M_{\pi}^{2}\right)}{s^{\ell+3}} \frac{(\ell+2)^{2}(\ell+1)}{\ell+3 / 2}\right\},
\end{aligned}
$$

where $F^{I}(s, t)=T^{I}(s, t) / 4 \pi^{2}$ and the primes denote the derivative with respect to $\cos \theta$. This formula allow us to calculate the $c$ parameters for the P, D0, D2 and F wave. For the S waves we provide two new sum rules, and one for $c_{P}$, in order to reduce its error:

$$
\begin{aligned}
c_{S 2}= & -6 b_{P}-10 a_{D 2}+\frac{8}{M_{\pi}} \int_{4 M_{\pi}^{2}}^{\infty} d s\left\{\frac{\operatorname{Im} F^{0+}(s, 0)}{s^{3}}\right. \\
+ & \left.\frac{1}{\left(s-4 M_{\pi}^{2}\right)^{5 / 2}}\left[\frac{\operatorname{Im} F^{0+}(s, 0)}{\sqrt{s-4 M_{\pi}^{2}}}-\frac{2 M_{\pi} a_{S 2}^{2}}{\pi}-\frac{s-4 M_{\pi}^{2}}{\pi}\left(\frac{M_{\pi}}{2}\left(2 a_{S 2} b_{S 2}+a_{S 2}^{4}\right)-\frac{a_{S 2}^{2}}{4 M_{\pi}}\right)\right]\right\} \\
c_{S 0}= & -2 c_{S 2}-20 a_{D 2}-10 a_{D 0}+\frac{12}{M_{\pi}} \int_{4 M_{\pi}^{2}}^{\infty} d s\left\{\frac{\operatorname{Im} F^{00}(s, 0)}{s^{3}}+\frac{1}{\left(s-4 M_{\pi}^{2}\right)^{5 / 2}}\left[\frac{\operatorname{Im} F^{00}(s, 0)}{\sqrt{s-4 M_{\pi}^{2}}}\right.\right. \\
& \left.\left.-\frac{4 M_{\pi}\left(2 a_{S 2}^{2}+a_{S 0}^{2}\right)}{3 \pi}-\frac{s-4 M_{\pi}^{2}}{3 \pi}\left(M_{\pi}\left[2\left(2 a_{S 2} b_{S 2}+a_{S 2}^{4}\right)+2 a_{S 0} b_{S 0}+a_{S 0}^{4}\right]-\frac{2 a_{S 2}^{2}+a_{S 0}^{2}}{2 M_{\pi}}\right)\right]\right\} \\
c_{P}= & -\frac{14 a_{F}}{3}+\frac{16}{3 M_{\pi}} \int_{4 M_{\pi}^{2}}^{\infty} d s\left\{\frac{\operatorname{Im} F^{I=0}(s, 0)}{3 s^{4}}-\frac{\operatorname{Im} F^{I=1}(s, 0)}{2 s^{4}}\right. \\
& \left.-\frac{5 \operatorname{Im} F^{I=2}(s, 0)}{6 s^{4}}+\left[\frac{\operatorname{Im} F^{I=1}(s, 0)}{\left(s-4 M_{\pi}^{2}\right)^{4}}-\frac{3 a_{P}^{2} M_{\pi}}{4 \pi\left(s-4 M_{\pi}^{2}\right)^{3 / 2}}\right]\right\}
\end{aligned}
$$

The derivation is similar to the sum rules obtained for $b_{P}, b_{S 0}$ and $b_{S 2}$ in [15]. They correspond to the threshold limit, taken from above, of the second derivative of a forward dispersion relation for the $F^{I=1}, F^{0+}$ and $F^{00}$ amplitudes, respectively. Let us recall that $F^{0+}=F^{I=2} / 2+F^{I=1} / 2$ whereas $F^{00}=2 F^{I=2} / 3+F^{I=0} / 3$. For a list of the resulting values, we refer the reader to Table II of the original reference [11]. 
Determination of ChPT low energy constants from a precise description of $\pi$ - $\pi$ scattering threshold parameters Jenifer Nebreda

\begin{tabular}{lccccc}
\hline \hline Fit to & $\bar{l}_{1}$ & $\bar{l}_{2}$ & $\bar{l}_{3}$ & $\bar{l}_{4}$ & $\chi^{2} /$ d.o.f. \\
\hline$a_{S}, b_{S}, a_{P}$ & $1.1 \pm 1.0$ & $5.1 \pm 0.7$ & $-1 \pm 8$ & $7.1 \pm 0.7$ & 0.23 \\
$a_{D}$ & $-1.75 \pm 0.22$ & $5.91 \pm 0.10$ & - & - & 0 \\
$c_{S}$ & $-2.4 \pm 0.9$ & $4.8 \pm 0.4$ & - & - & 0 \\
$a_{S}, b_{S}, a_{P}, a_{D}, c_{S}, b_{P}$ & $-2.06 \pm 0.14$ & $5.97 \pm 0.07$ & $-5 \pm 8$ & $7.1 \pm 0.6$ & 7.9 \\
$a_{S}, b_{S}, a_{P}, a_{D}, c_{S}, b_{P}$, using $f_{0}$ & $-1.06 \pm 0.11$ & $4.6 \pm 0.9$ & $0 \pm 6$ & $5.0 \pm 0.3$ & 7.06 \\
\hline Estimate $O\left(p^{4}\right)$ & $-1.5 \pm 0.5$ & $5.3 \pm 0.7$ & $-3 \pm 7$ & $6.0 \pm 1.2$ & - \\
\hline
\end{tabular}

Table 1: $O\left(p^{4}\right)$ fits to different sets of threshold parameters containing polynomial $O\left(p^{4}\right)$ contributions. We observe that a precise description of the observables is not possible at one loop. Anyway, we provide an estimate of how much one should enlarge the uncertainties of the LECs if, for simplicity, one still insists in using the one-loop formalism.

\section{3. $O\left(p^{4}\right)$ fits}

We start by fitting the threshold parameters using the one-loop ChPT amplitudes, which will help us check the stability of the LECs values and the need for higher order counterterms.

Let us recall that to $O\left(p^{4}\right)$ only four LECs appear in $\pi \pi$ scattering, customarily denoted by $\bar{l}_{1}, \ldots, \bar{l}_{4}$, which are basically the $l_{i}^{r}(\mu)$ at the $\mu=M_{\pi}$ scale and normalized so that they have values of order one [2]. Note, however, that $\bar{l}_{3}$ and $\bar{l}_{4}$ only appear through the quark mass dependence of $M_{\pi}$ and $f_{\pi}$, respectively, and therefore we cannot expect much sensitivity to these two parameters from fits to the coefficients of the momentum expansion of amplitudes.

In addition, since the LECs only appear in the polynomial part of the partial waves, which at one loop is of $O\left(p^{4}\right)$, only ten observables carry any dependence on the LECs: $a_{S(0,2)}, a_{P}, b_{S(0,2)}$, $b_{P}, c_{S(0,2)}$ and $a_{D(0,2)}$. The rest of the coefficients multiply powers of the momentum higher than $p^{4}$ and thus, do not receive a contribution from the $O\left(p^{4}\right)$ LECs.

In Table 1 we show the results of our fits. First, we have fitted only the observables whose leading contribution is of $O\left(p^{2}\right)$, since these might be more stable under the higher order corrections. The fit comes out with relatively low $\chi^{2} /$ d.o.f. Next we have presented a determination of $\bar{l}_{1}$ and $\bar{l}_{2}$, which can be fixed from $a_{D 0}$ and $a_{D 2}$ alone. It is evident that the resulting values from those two fits are incompatible. The incompatibility is even worse when fitting simultaneously the ten observables that depend on $\bar{l}_{i}$ to $O\left(p^{4}\right)$. Finally, the effect of higher order corrections has been studied by fitting to the one-loop amplitude but replacing $f_{\pi}$ by $f_{0}$ in the $O\left(p^{4}\right)$ terms, since the two expressions only differ in higher order contributions. This we show in row 5 of Table 1. The $\chi^{2} /$ d.o.f is somewhat lower, but the values of the LECs come out rather different from the previous calculation.

These results imply that, to the present level of precision, the one-loop ChPT formalism is not enough and calls for higher order corrections. If one still wants to use this simpler version instead of the full two-loop amplitude one can include the effect of higher orders into a systematic uncertainty of the LECs. We propose to take the weighted average of the two previous fits, including a systematic uncertainty to cover the LECs values of both fits. In Table IV of the original reference [11], we compare the resulting threshold parameters obtained using this averaged set with 
the experimental values calculated as explained in the previous section. Thanks to the larger uncertainty, the threshold parameters obtained are compatible within errors with the experimental values, except for $b_{S 0}$ and $b_{P}$, which differ by more than three and two standard deviations respectively.

\section{4. $O\left(p^{6}\right)$ fits}

As already commented in the introduction, the two-loop $\pi \pi$ scattering amplitudes can be recast in terms of six independent terms multiplied by their corresponding low energy constants $\bar{b}_{i}$. In turn, these $\bar{b}_{i}$ can be rewritten in terms of the four $O\left(p^{4}\right)$ LECs and six combinations $r_{i}$ of the $O\left(p^{6}\right)$ LECs. The difference between writing the amplitude in one way or the other is $O\left(p^{8}\right)$. However, despite increasing the number of parameters to ten, the $O\left(p^{6}\right)$ amplitude still provides just six independent structures. As a consequence, the fits in terms of $\bar{l}_{i}$ and $r_{i}$ are much more unstable, and can even lead to spurious solutions. For this reason we focus on the fits in terms of $\bar{b}_{i}$, and refer to the appendix of the original reference [11] for a study of the $\bar{l}_{i}, r_{i}$ fits.

We first fit the ten threshold parameters used in the previous section because, having a nonzero $O\left(p^{4}\right)$ polynomial contribution, we expect these to be more stable under higher order ChPT corrections. In the first row of Table 2 we show the resulting $\bar{b}_{i}$ for this fit, which describes fairly well the fitted observables with a $\chi^{2} /$ d.o.f. $=1.2$.

However, when fitting all 18 threshold parameters, we obtain somewhat different LECs (see the second row of Table 2) and the $\chi^{2} /$ d.o.f. comes out rather poor. We have noticed that $c_{P}$ alone contributes almost to one third of the total $\chi^{2}$. This might indicate that $c_{P}$ receives important higher order contributions that are not being taken into account in the $O\left(p^{6}\right)$ calculation. Once again we obtain a crude estimate of the size of higher order ChPT corrections, by changing $f_{\pi}$ by $f_{0}$ in the last term of the expansion. $c_{P}$ suffers indeed the largest change, by almost $80 \%$.

Thus, we proceed to fit again all threshold parameters except $c_{P}$. The result is shown in the third row of Table 2. The fit quality improves sizably, but we still get a high $\chi^{2} /$ d.o.f. $=2.9$, which indicates that the two-loop calculation may not be enough to describe even the remaining threshold parameters with their current level of precision.

Again, we see the effect of higher order corrections by making a fit replacing $f_{\pi}$ by $f_{0}$ in the $O\left(p^{6}\right)$ terms. We show the results in the fourth row of Table 2. Surprisingly, we now obtain a good $\chi^{2} /$ d.o.f. $=1.0$ and all LECs are less than two standard deviations away from those obtained by fitting only the threshold parameters with an $O\left(p^{4}\right)$ polynomial part. We conclude that, by excluding $c_{P}$, the two-loop fit can give an acceptable description of the rest of threshold parameters.

For this reason, we have once more made a weighted average of the two fits (the one using $f_{\pi}$ and the one using $f_{0}$ ) adding systematic uncertainties to cover both sets. This we show in the fifth row of Table 2, where we can see that they are also quite compatible with previous determinations in the literature [10].

\section{Summary}

We have reported a work [11] on the determination the low energy constants of $S U(2)$ Chiral Perturbation Theory (ChPT) at one and two loops by fitting to the threshold parameters obtained 
Determination of ChPT low energy constants from a precise description of $\pi$ - $\pi$ scattering threshold parameters Jenifer Nebreda

\begin{tabular}{lccccccc}
\hline \hline Fit to & $\bar{b}_{1}$ & $\bar{b}_{2}$ & $\bar{b}_{3}$ & $\bar{b}_{4}$ & $\bar{b}_{5}$ & $\bar{b}_{6}$ & $\frac{\chi^{2}}{\text { d.o.f. }}$ \\
\hline$a_{S}, b_{S}, a_{P}, a_{D}, c_{S}, b_{P}$ & $-14 \pm 4$ & $14.6 \pm 1.2$ & $-0.29 \pm 0.05$ & $0.76 \pm 0.02$ & $0.1 \pm 1.1$ & $2.2 \pm 0.2$ & 1.2 \\
All & $-2 \pm 3$ & $14.2 \pm 1.0$ & $-0.39 \pm 0.04$ & $0.746 \pm 0.013$ & $3.1 \pm 0.3$ & $2.58 \pm 0.12$ & 5.2 \\
All but $c_{P}$ & $-6 \pm 3$ & $15.9 \pm 1.0$ & $-0.36 \pm 0.04$ & $0.753 \pm 0.013$ & $2.2 \pm 0.4$ & $2.44 \pm 0.12$ & 2.9 \\
All but $c_{P}$, using $f_{0}$ & $-12 \pm 3$ & $13.9 \pm 0.9$ & $-0.30 \pm 0.04$ & $0.726 \pm 0.013$ & $1.0 \pm 0.3$ & $1.93 \pm 0.08$ & 1.04 \\
\hline Estimate $O\left(p^{6}\right)$ & $-10.5 \pm 5.1$ & $14.5 \pm 1.8$ & $-0.31 \pm 0.06$ & $0.73 \pm 0.02$ & $1.3 \pm 1.0$ & $2.1 \pm 0.4$ & - \\
\hline Ref. [10] & $-12.4 \pm 1.6$ & $11.8 \pm 0.6$ & $-0.33 \pm 0.07$ & $0.74 \pm 0.01$ & $3.6 \pm 0.4$ & $2.35 \pm 0.02$ & -
\end{tabular}

Table 2: $O\left(p^{6}\right)$ fits. In the first row we only fit to observables containing polynomial $O\left(p^{4}\right)$ contributions. Next we show the fit to all the threshold parameters obtained in this work. The quality is rather poor, but most of the disagreement is caused by $c_{P}$. When this observable is omitted, the resulting fits are much better, specially when using $f_{0}$ instead of $f_{\pi}$ in the last term of the ChPT expansion. We provide an estimate of the LECs uncertainties from the fits to all observables except $c_{P}$, as a weighted average of the fits using $f_{0}$ or $f_{\pi}$. The resulting $\bar{b}_{i}$ parameters are very consistent with previous determinations, listed in the last row.

from sum rules using a recent and precise dispersive analysis of data [12], together with six additional observables that we have studied here.

We have checked that the one-loop formalism is clearly insufficient to accommodate the present level of precision. The $\chi^{2} / d$.o.f. improves remarkably when using the two-loop expansion, although it is still not sufficient to get a good quality fit. This suggests that even higher order ChPT contributions may still be required to describe all these observables simultaneously.

\section{References}

[1] S. Weinberg, Physica A96 (1979) 327.

[2] J. Gasser and H. Leutwyler, Annals Phys. 158 (1984) 142;

[3] M. Knecht, B. Moussallam, J. Stern, N. H. Fuchs, Nucl. Phys. B457, 513-576 (1995).

[4] J. Bijnens, G. Colangelo, G. Ecker, J. Gasser, M. E. Sainio, Phys. Lett. B374, 210-216 (1996); Nucl. Phys. B508, 263-310 (1997) [Erratum-ibid. B 517, 639 (1998)].

[5] G. Colangelo et al., Eur. Phys. J. C 71, 1695 (2011).

[6] D. Espriu, E. de Rafael, J. Taron, Nucl. Phys. B345, 22-56 (1990). F. J. Llanes-Estrada, P. de A. Bicudo, Phys. Rev. D68, 094014 (2003).

[7] G. Ecker, J. Gasser, A. Pich, E. de Rafael, Nucl. Phys. B321, 311 (1989). J. F. Donoghue, C. Ramirez, G. Valencia, Phys. Rev. D39 (1989) 1947.

[8] C. Riggenbach, J. Gasser, J. F. Donoghue and B. R. Holstein, Phys. Rev. D 43, 127 (1991);

[9] G. Amoros, J. Bijnens and P. Talavera, Nucl. Phys. B 585, 293 (2000) [Erratum-ibid. B 598, 665 (2001)];

[10] G. Colangelo, J. Gasser and H. Leutwyler, Nucl. Phys. B 603, 125 (2001).

[11] J. Nebreda, J. R. Pelaez and G. Rios, arXiv:1205.4129 [hep-ph].

[12] R. Garcia-Martin, R. Kaminski, J. R. Pelaez, J. Ruiz de Elvira, F. J. Yndurain, Phys. Rev. D83, 074004 (2011).

[13] J. R. Batley et al. [ NA48-2 Collaboration ], Eur. Phys. J. C70, 635-657 (2010).

[14] S. R. Beane et al. [NPLQCD Collaboration], Phys. Rev. D 85, 034505 (2012).

[15] J. R. Pelaez and F. J. Yndurain, Phys. Rev. D 71, 074016 (2005); R. Kaminski, J. R. Pelaez and F. J. Yndurain, Phys. Rev. D 77, 054015 (2008) 